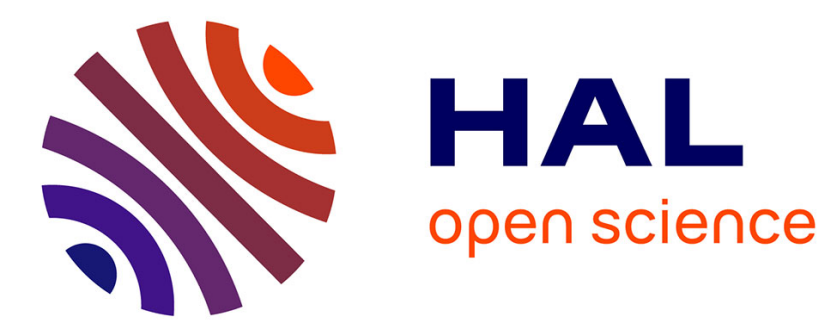

\title{
From stereoscopic images to semi-regular meshes
}

\author{
Jean-Luc Peyrot, Frédéric Payan, Marc Antonini
}

\section{To cite this version:}

Jean-Luc Peyrot, Frédéric Payan, Marc Antonini. From stereoscopic images to semi-regular meshes. Signal Processing: Image Communication, 2016, 40, pp.97-110. 10.1016/j.image.2015.11.004 . hal01236999

\section{HAL Id: hal-01236999 \\ https://hal.science/hal-01236999}

Submitted on 8 Dec 2015

HAL is a multi-disciplinary open access archive for the deposit and dissemination of scientific research documents, whether they are published or not. The documents may come from teaching and research institutions in France or abroad, or from public or private research centers.
L'archive ouverte pluridisciplinaire HAL, est destinée au dépôt et à la diffusion de documents scientifiques de niveau recherche, publiés ou non, émanant des établissements d'enseignement et de recherche français ou étrangers, des laboratoires publics ou privés. 


\title{
From stereoscopic images to semi-regular meshes
}

\author{
Jean-Luc Peyrot, Frédéric Payan and Marc Antonini \\ Laboratory I3S - University Nice - Sophia Antipolis and CNRS (France) - UMR 7271
}

\begin{abstract}
The pipeline to get the semi-regular mesh of a specific physical object is long and fastidious : physical acquisition (creating a dense point cloud), cleaning/meshing (creating an irregular triangle mesh), and semi-regular remeshing. Moreover, these three stages are generally independent, and processed successively by different tools. To overcome this issue, we propose in this paper a new framework to design semi-regular meshes directly from stereoscopic images. Our semi-regular reconstruction technique first creates a base mesh by using a feature-preserving sampling on the stereoscopic images. Afterwards, this base mesh is passed to a coarse-to-fine meshing process to get the semi-regular mesh of the original surface. Experimental results prove the reliability and the accuracy of our approach in terms of shape fidelity, compactness, but also runtime, since many steps have been parallelized on the GPU.
\end{abstract}

Keywords: Semi-regular mesh, 3D reconstruction, stereoscopy, acquisition, Poisson-disk sampling, GPU.

\section{Introduction}

Motivated by the high fidelity and the realism of the numerical models, and supported by the increasing storage capacities, the acquisition devices provide now high resolution meshes, ensuring the preservation of the finest details. Consequently these data are massive, and cannot be easily managed by any workstation or mobile device with limited memory and bandwidth. The semiregular meshes are a good way to overcome these issues, because of their sca8 lability and their compactness. Indeed, the semi-regular meshes are based on 

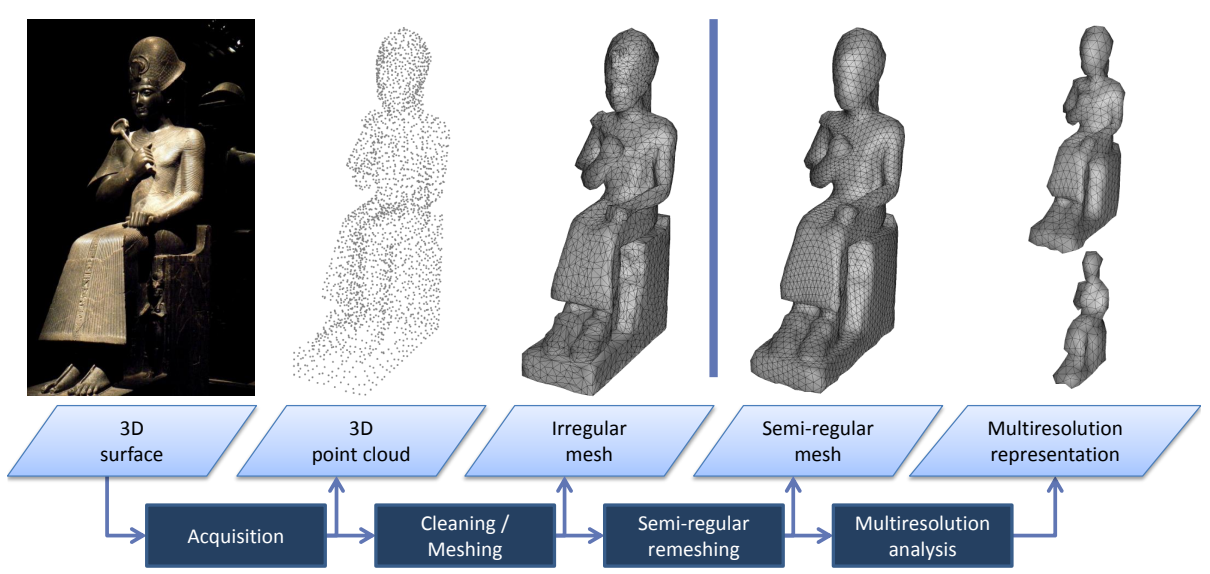

Figure 1: The pipeline to get a semi-regular mesh from a physical object, and its application to multiresolution analysis.

9 a regular subdivision connectivity, well-suited to display or transmit a mesh at different levels of details. This subdivision connectivity also allows a compact representation since only the connectivity of the lowest level of details is needed to reconstruct the full connectivity. This semi-regular structure is also adapted to multiresolution analysis (Lounsbery et al., 1997) and wavelet compression (Payan and Antonini, 2006). Despite their good properties, the semi-regular meshes are sometimes forsaken by users because they are not provided by current acquisition systems which only provide point clouds. So, if one wants to produce a semi-regular mesh of a specific physical object, the pipeline presented in figure 1 must be processed : physical acquisition (creating a dense point cloud), cleaning/meshing (removing redundant points and noise inherent to acquisition process, and creating an irregular triangle mesh), and then semiregular remeshing (Payan et al., 2015). This pipeline is long and fastidious, especially as these three stages are performed independently.

Our original idea is to make the design of semi-regular meshes easier, by simplifying the classical pipeline shown above. This paper, that is an extended version of (Peyrot et al. 2014), presents a coarse-to-fine approach that allows an acquisition system to provide semi-regular meshes as output, thus avoiding a 
${ }_{27}$ remeshing process. We focused on stereoscopic systems, because stereoscopy is

28 an increasing field of interest in surface reconstruction, due to its rapidity and accuracy.
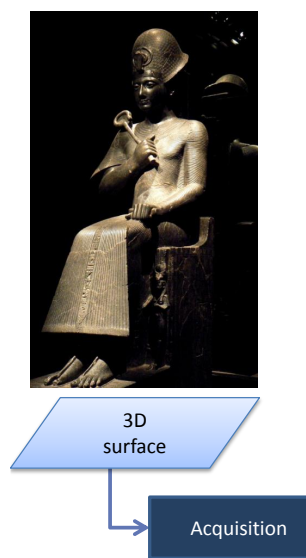
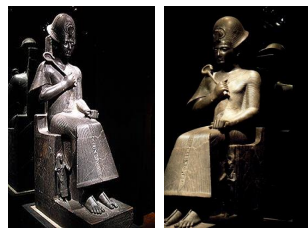

Stereoscopic images

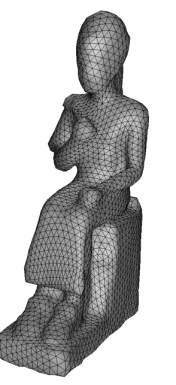

Semi-regular mesh

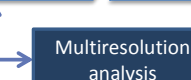

FIGURE 2: Our 3D reconstruction technique that produces a semi-regular mesh directly from stereoscopic images.

Our method, depicted in figure 2, relies on an analysis of the stereoscopic images to get a base mesh that captures the salient features of the original object, followed by a coarse-to-fine meshing that generates the semi-regular output. The most innovative part of our algorithm is the use of the stereoscopic images as parameterization domain to create the semi-regular mesh.

The remaining of the paper is organized as follows. In Section 2 we remind the reader of the basics of semi-regular meshes and briefly review two prior methods of surface reconstruction based on stereoscopy and parameterization. Section 3 presents our semi-regular reconstruction method. Experimental results are presented in Section 4 Finally, Section 5 summarizes our contributions, and proposes future work. 


\section{Background}

44

45

46 fours down to a lower resolution mesh.

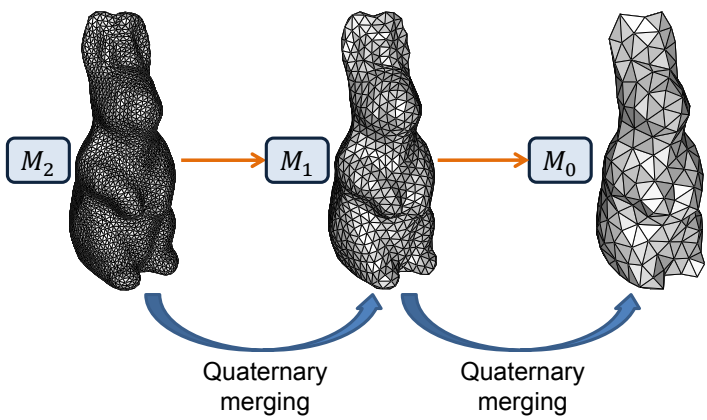

Figure 3: Semi-regular mesh of the model RABBit ( $L=3$ levels of resolutions).

47

\subsection{Semi-regular meshes}

A semi-regular mesh $M_{s r}$ is a structured mesh defined by $L$ levels of resolutions (figure 3), where all the triangles at a specific level can be merged by

This merging process can be applied $(L-1)$ times to $M_{s r}$ until obtaining a base mesh $M_{0}$ that represents the lowest resolution of $M_{s r}\left(M_{s r}\right.$ can be seen as $\left.M_{L-1}\right)$. A semi-regular mesh is sometimes called a subdivision mesh, because a subdivision scheme is applied on the mesh at resolution $l$ to generate the semi-regular mesh at the finer level of resolution $(l+1)$.

\subsection{Presentation of two prior surface reconstruction methods}

We now present two prior reconstruction methods similar to our proposal, because they are based on multi-view images and use a parameterization. Interested reader will find a complete presentation of general reconstruction methods in (Seitz et al. 2006).

The method proposed in (Park et al., a) combines the advantages of geometric and photometric techniques, thanks to the surface parameterization. It consists in associating a Multi-View Stereo (MVS) reconstruction process that relies on a correspondence between pixels from different multi-view images, and a Shape from Shading method that utilizes the surface reflectance. The authors 

to provide different lighting configurations.

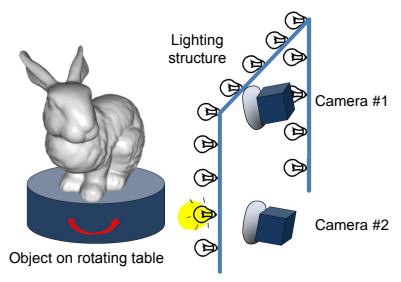

Figure 4: Image acquisition system presented in (Park et al. a) (image of (Park et al. a)). leading to high-quality reconstructions.

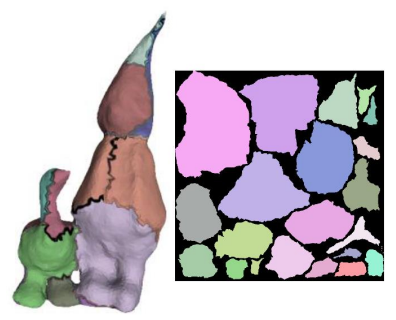

Figure 5: Charts defined on the base mesh, and its associated parameterization (image of (Park et al. a) ).

First of all, the technique Structure from Motion (Snavely et al., 2006) generates the 3D point cloud of the scanned object. The multi-view method $M V S$ of (Hernandez et al. 2008) is then used to generate a depth-map, and the base mesh. The third step consists in creating a parameterization by charts (Zhou et al. 2004) (see figure 5). Finally, from the parameterization and the normals estimated at each vertex of the base mesh, a refinement procedure is applied,

Another relevant approach is proposed in (Pietroni et al., 2011). The authors present a quadrangular remeshing technique based on a global and low distortion parameterization of different kinds of surfaces (polygonal meshes, point 
clouds...). The principle, illustrated in figure 6, is to first generate a set of distance maps $U^{i}$ of the input data. Then, each image $U^{i}$ is parameterized into a 2D planar domain, while controlling the resulting distortion at the frontiers of the images in the final parameterization. Finally, a sampling in the parameterization domain creates a quadrangular semi-regular mesh.

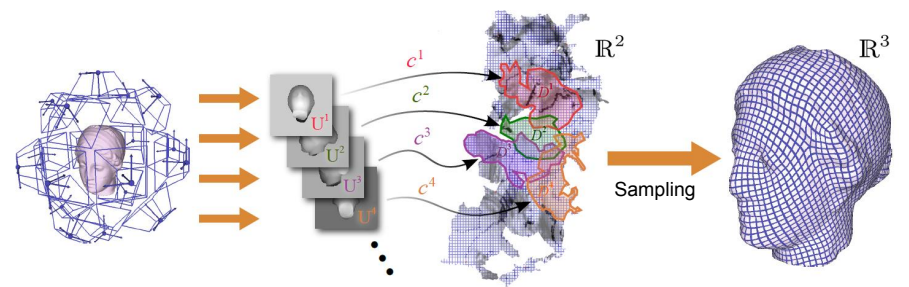

Figure 6: Overview of (Pietroni et al. 2011)'s method (image of (Pietroni et al., 2011)).

Discussion These two parameterization-based methods are reliable. However, we cannot refer to (Park et al. a) to get a semi-regular mesh directly from stereoscopic images, as a coarse 3D mesh must be built before creating the parameterization. The other method, (Pietroni et al. 2011), is closely related to our goal, it requires a cross-field technique that might be complex and uses triangles embedded in $\mathbb{R}^{3}$. A contrario, our method strives to minimize the use of the $3 \mathrm{D}$ connectivity by using the stereoscopic images as parameterization domain and a coarse-to-fine approach.

\section{Presentation of our semi-regular reconstruction method}

\subsection{Overview}

To highlight the interest of our approach, we first present the classical pipeline to get a semi-regular mesh of a physical object with a stereoscopic system (Figure 7(a).

1. Stereo matching The goal is to find the Pixels Of Interest (POI) region in the two images that represents the physical object (Scharstein and Szeliski, 2002). The POI region gathers the couples of pixels that correspond 
to a same point in the 3D space through both cameras (yellow parts on the left and right stereoscopic images). The POI region is only a subset of the stereoscopic images since it is impossible to capture the same set of $3 \mathrm{D}$ points from two different points of views.

2. 3D coordinates computation The coordinates of the $3 \mathrm{D}$ points are computed for all the pixels belonging to the POI region (Hartley and Zisserman, 2004). These two first steps are done by the acquisition system.

3. Cleaning/meshing The 3D point cloud must be cleaned, and then triangulated, leading to a dense irregular mesh. This is the second independent process.

4. Simplification The semi-regular remeshing can now be done (third independent process) : the irregular mesh is first simplified to obtain a coarse mesh corresponding to the base mesh of final semi-regular mesh. During this stage, a parameterization of the irregular mesh vertices is generally computed onto this base mesh.

5. Refinement The base mesh is subdivided several times ( $1: 4$ subdivision) to create the different resolutions of the final semi-regular mesh. Generally, the aforementioned parameterization optimizes the positioning of the new vertices added by subdivision.

The originality of our semi-regular reconstruction method, illustrated in figure $7(\mathrm{~b})$ is that it mainly works onto the $2 \mathrm{D}$ domain defined by the stereoscopic images, and thus can be included in the acquisition system :

1. Stereo matching This stage is identical to the one in the classical approach.

2. POI pixel classification The goal is to detect the feature lines in the POI region. The creation of the base mesh will be guided by these feature lines to ensure that the geometrical features are preserved on the final semi-regular output. Moreover, such assertion greatly improves the reconstruction quality. 


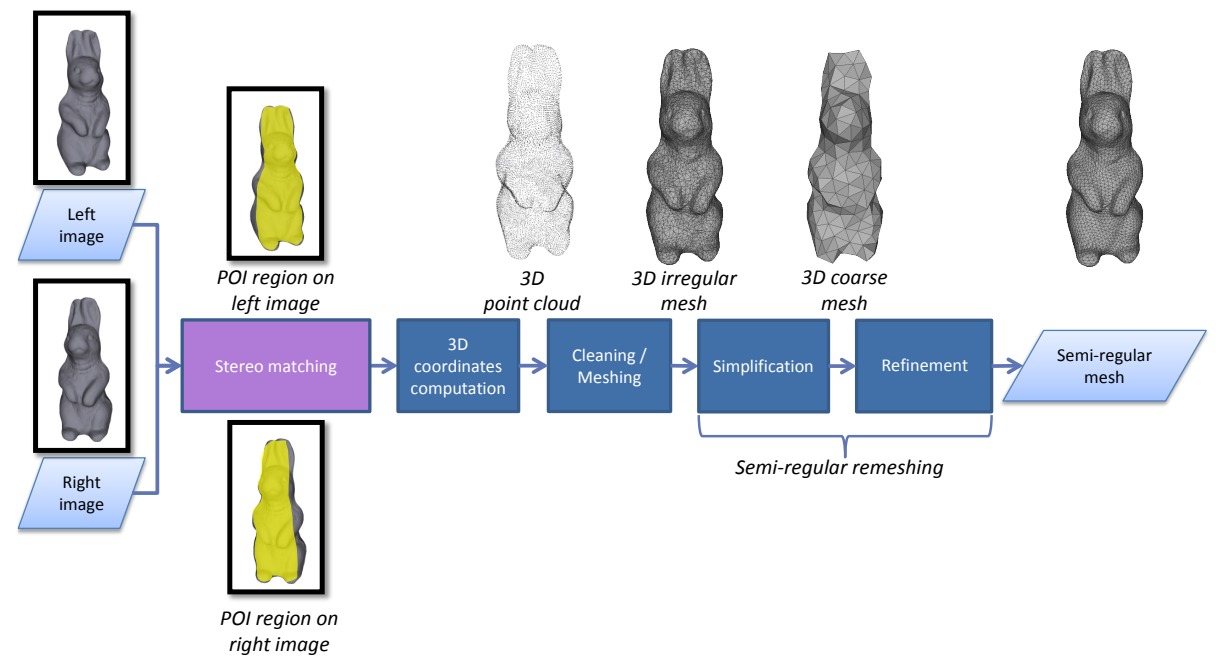

(a) Classical pipeline of semi-regular reconstruction.

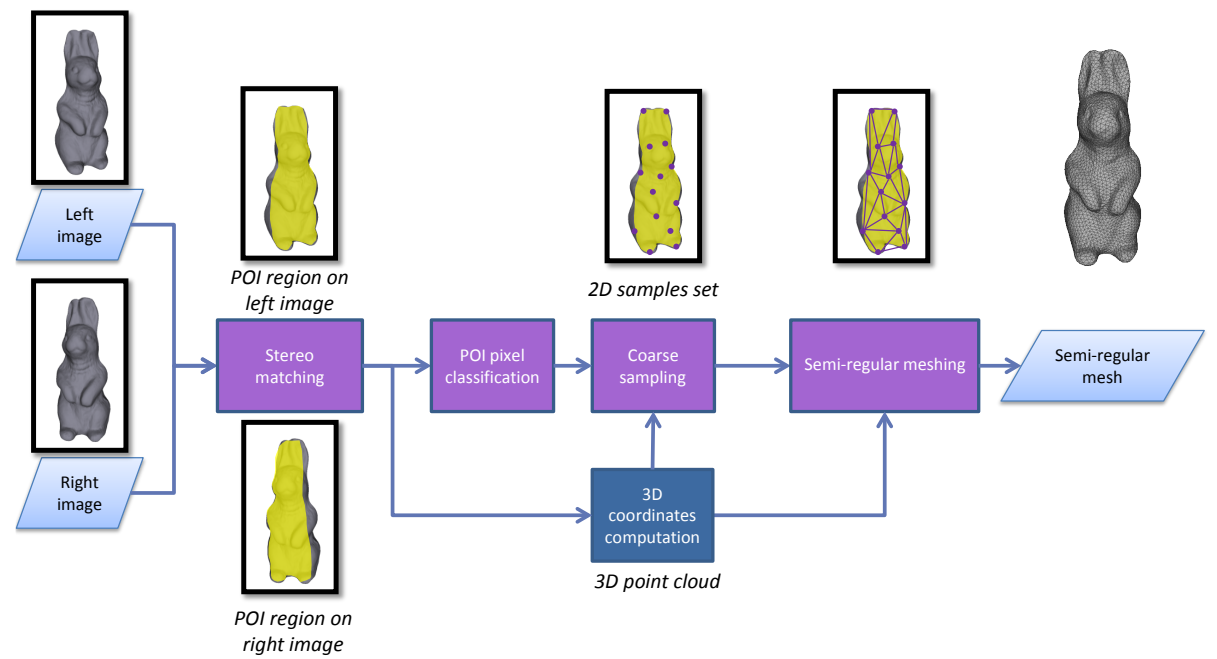

(b) Proposed semi-regular reconstruction method.

Figure 7: How to get a semi-regular mesh from stereoscopic images? The classical pipeline (top) Vs our direct coarse-to-fine reconstruction method (down). Purple and blue blocks indicate that the process is realized in $2 \mathrm{D}$ and $3 \mathrm{D}$ space, respectively. 
3. Coarse sampling A coarse sampling, constrained by the feature lines, is done to retrieve a set of $2 \mathrm{D}$ samples that will be later the vertices of the base mesh. This stage is based on 2D Poisson-disk sampling, to ensure a good distribution of the samples over the POI region.

4. Semi-regular meshing The set of $2 \mathrm{D}$ samples is first triangulated to obtain a $2 \mathrm{D}$ base mesh. Then, this $2 \mathrm{D}$ base mesh is subdivided several times, to get a 2D semi-regular mesh of the POI region. Finally, the 3D semi-regular mesh is obtained by computing the $3 \mathrm{D}$ coordinates associated to the $2 \mathrm{D}$ samples.

\subsection{POI pixel classification}

To detect the feature lines in the POI region, we first classify the pixels according to their curvature values ${ }^{1}$, as described below.

A tensor $T_{p(u, v)}$ is calculated at each pixel $p(u, v)$ in the POI region using

$$
T_{p(u, v)}=\sum_{u^{\prime}=u-n}^{u^{\prime}=u+n} \sum_{v^{\prime}=v-n}^{v^{\prime}=v+n} \overrightarrow{N^{\prime}} \cdot \overrightarrow{N^{\prime}} t
$$

where $\overrightarrow{N^{\prime}}$ is the $3 \mathrm{D}$ normal associated to the neighbor pixel $p^{\prime}\left(u^{\prime}, v^{\prime}\right)$, and $n$ depends on the size of the considered neighbor region of $p$. The three eigenvalues of $T_{p(u, v)}$ are then computed with the Jacobi operator, and a thresholding operation performs the segmentation of high curvature area. In order to reduce the runtime and benefit the independence of the operation at each pixel $p$, this classification is GPU-parallelized.

However, this classification is not precise enough to be exploited as it is. A parallelized thinning technique (Zhang and Suen, 1984) is thus applied to the 'high curvature' pixels to finely detect the sharp edges. Thinning a set of

1. In the current version, the curvature values are calculated with the technique of $($ Park et al. b) from the 3D normals associated to the corresponding 3D point cloud. In fine, to limit the use of $3 \mathrm{D}$ information, this technique will be replaced in our algorithm by the recent technique of (DTA) that computes the 3D normals directly from stereoscopic images. 
neighbor pixels consists in generating a skeleton (i.e. a set of median lines) that presents the same topology as the related shape.

Once the thinning is done, we classify the pixels in the POI region according to three classes :

- corners containing the pixels where the median lines intersect in the image ;

- sharp features containing the remaining skeleton pixels;

- smooth regions containing the other pixels.

Figure 8 depicts several results of classification obtained with this method.

This classification of POI pixels will help the subsequent sampling to preserve geometrical features and thus to provide a consistent base mesh in terms of global shape and geometrical characteristics, as explained below.

\subsection{Coarse sampling}

This stage is inspired by the feature-preserving Poisson-disk sampling for surfaces of (Peyrot et al. 2015), which is based on a dart throwing.

This approach can be efficiently adapted to our setting : the sampling domain $\Omega$ becomes the POI region of the stereoscopic images (instead of a surface mesh in (Peyrot et al. 2015) , and the feature lines detected by the previous stage guide the distribution of $2 \mathrm{D}$ samples.

However, as the output 2D samples of this stage will define the vertices of the base mesh, they must be consistently distributed over the surface of the object (and not especially over its stereoscopic images). Therefore the sampling is done onto the POI region, to benefit from its implicit $2 \mathrm{D}$ connectivity, but the distances between samples are computed in the 3D space with Dijkstra's algorithm (Dijkstra, 1959).

The principle of the dart throwing on a $2 \mathrm{D}$ image is the following : i) one pixel in the $2 \mathrm{D}$ domain $\Omega$ is chosen randomly, ii) a disk is computed around it, according to a radius $R$ that depends on the target number $N$ of samples and a density function, iii) this pixel is considered as a valid sample if the disk 


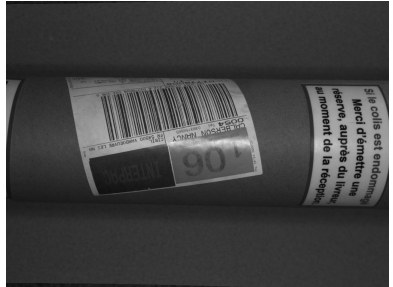

(a)

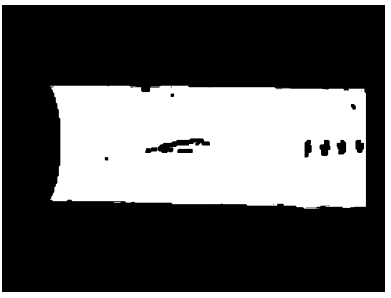

(d)

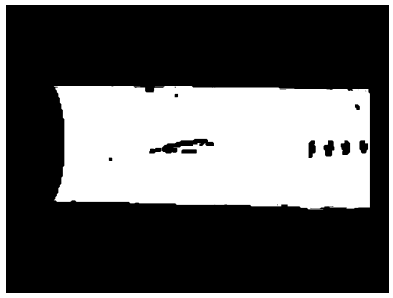

$(\mathrm{g})$

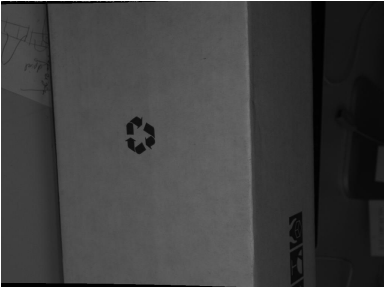

(b)

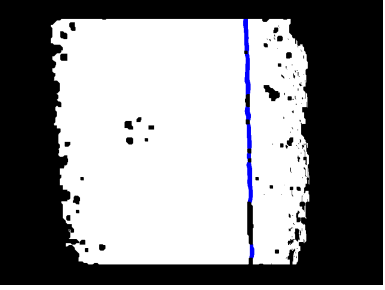

(e)

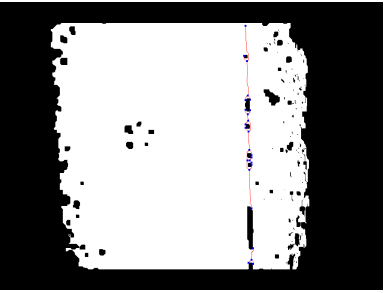

(h)

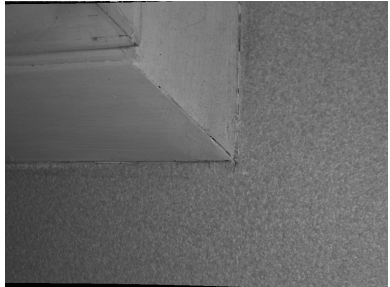

(c)

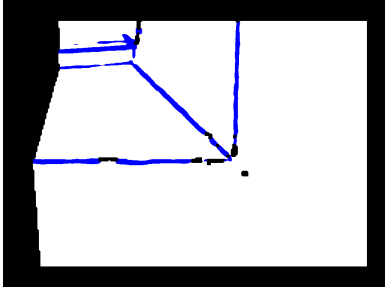

(f)

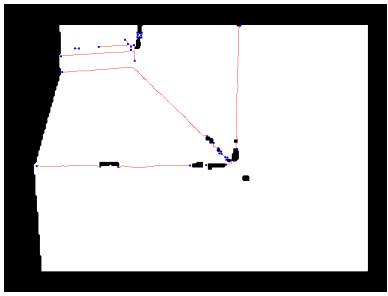

(i)

Figure 8: Detection of the feature lines via our classification technique. First row : left stereoscopic images obtained with our scanner (models Pipe, Box and WALL). Second row : detection of high curvature areas (in blue). Third row : resulting classification after thinning : white, red and blue pixels represent respectively the smooth regions, the sharp features and the corners. 
does not intersect the disks relative to the samples already accepted (ensuring a minimal distance between the samples).

One key idea of the proposed sampling technique is the computation of the radius $R$ onto the surface in the $3 \mathrm{D}$ space, while handling the POI region of the stereoscopic images. Given the requested number of samples $N$, we first calculate the horizontal $\delta_{i}$ and vertical $\delta_{j}$ deviations between samples when a uniform sampling pattern is realized on the stereoscopic image. It generates a grid of samples of dimension $\mathbb{N}_{\delta_{i}} \times \mathbb{N}_{\delta_{j}}$, as depicted in figure 9 , where $N_{i}$ and $N_{j}$ represent the number of samples per row and per column, respectively $\left(N_{i} \times N_{j}=N\right)$.

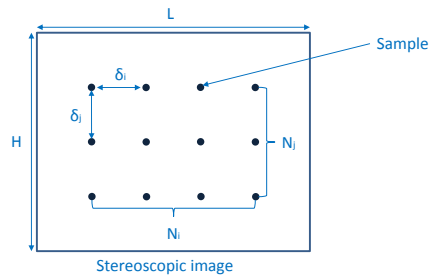

FiguRE 9: Example of uniform sampling performed on one stereoscopic image.

To take care of the fact that the sampling domain $\Omega$ is restricted to the pixels in the POI region, the distances $\delta_{i}$ and $\delta_{j}$ between samples along each dimension, are shrunk by a factor $\frac{L \times H}{\operatorname{Card}\{P O I\}}$, with $\operatorname{Card}\{P O I\}$ the number of pixels in $\Omega$. A uniform sampling can be realized using

$$
R=\frac{1}{3} \cdot \max \left(\delta_{i}, \delta_{j}\right) \cdot S_{r},
$$

where $S_{r}$ is the spatial resolution of the scanner $(0.3 \mathrm{~mm}$ in our case). With some objects, it can be convenient to realize an adaptive sampling, to better preserve the geometrical features for instance. In that case, the radius will depend on the surface curvature according to the following equation (Peyrot et al., 2015) :

$$
R=\frac{1}{3} \cdot \max \left(\delta_{i}, \delta_{j}\right) \cdot S_{r} \cdot\left(1+e^{C \cdot \lambda_{2}}+e^{C \cdot \lambda_{3}}\right) .
$$

Empirically, we put $C=-8.0$ for the pixels of the class sharp features, and $C=-6.0$ for the pixels of the class smooth regions. $\lambda_{2}$ and $\lambda_{3}$ are the eigenvalues 
of the tensor $T_{p(u, v)}$ computed in section 3.2 . In this formulation, the corners pixels keep the minimum radius given by equation (2). To determine the disks associated to the samples in function of the radius $R$, we recall that we use Dijkstra's algorithm to compute geodesic distances between 3D points, while using the connectivity of the $2 \mathrm{D}$ sampling domain $\Omega$. Therefore, a disk does not depend on the Euclidean distance between two given 2D samples, but on the sum of the lengths of the 3D segments defined by the shortest path in the POI region, as shown in figure 10 . As output of this stage, we get a set of $2 \mathrm{D}$ samples, that ensures a good distribution of the vertices of the 3D base mesh all over the scanned surface.
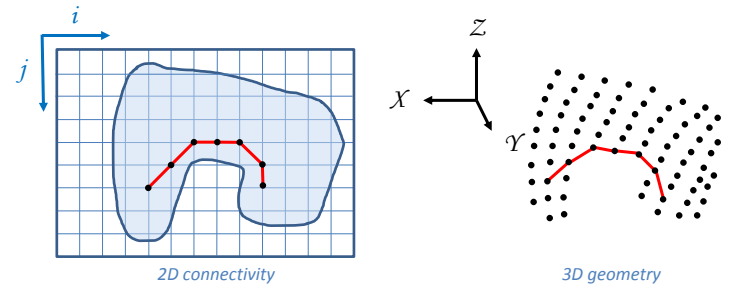

Figure 10: Computation of a geodesic distance between two points of the surface in $\mathbb{R}^{3}$ (right image), driven by the shortest path between the associated pixels in the POI region (light blue region, left image).

\subsection{Semi-regular meshing}

We now present how to generate a semi-regular mesh directly from the set of $2 \mathrm{D}$ samples defined previously. It is a three-stage process : creation of the $3 \mathrm{D}$ base mesh from the set of $2 \mathrm{D}$ samples, refinement by iterative subdivisions to get a 2D semi-regular mesh, and fitting in 3D space.

Creation of the base mesh. The base mesh is obtained via a constrained Voronoi relaxation (Lloyd, 1982) of the samples in the stereoscopic image domain, followed by the triangulation of the relaxed samples (via the dual of the Voronoi diagram (Rong et al. $)$ ). In our context, the Voronoi relaxation consists in first computing a Voronoi diagram of the pixels in function of the set of samples, and then displacing each sample to the centroid of its cell. This process is iteratively 
repeated until convergence. The relaxation greatly improves the mesh quality, when comparing with the triangulation that we could obtain directly from the initial voronoi diagram.

In this work, the Voronoi diagrams are generated with (Munshi et al., 2011), that is a GPU implementation of popular Dijkstra's algorithm (Dijkstra, 1959). We had to adapt this algorithm to process stereoscopic images. Moreover, to preserve the feature lines on the created base mesh, we added a constraint during the relaxation : the new samples must belong to the same class than the initial samples (corners, sharp features or smooth regions). In other words, if after relaxation a sample is moved to a pixel which does not belong to the same class, then the sample is displaced to the closest pixel of the same class. This technique is straightforward, but produces nice triangulations, while preserving geometrical features of the scanned object, as shown in figure 11. This figure shows also the poor triangulation obtained if the constraint is not included.
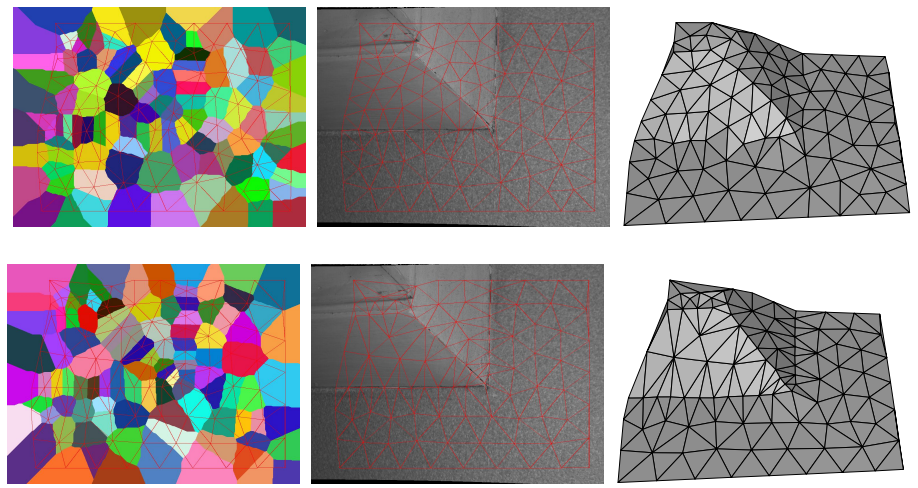

Figure 11: Base mesh generated by our Voronoi relaxation without (first row) or with (second row) the constraint on the feature lines. Left : final Voronoi diagram and triangulation. Middle : the same triangulation on the left stereoscopic image. Right : the resulting 3D base mesh.

Refinement. A 2D semi-regular mesh is first obtained by applying several midpoint subdivisions (Chen and Prautzsch, 2012) to the base mesh of the left stereoscopic image (see figure 12). Then, the surface fitting will embed the semi- 
regular mesh in the $3 \mathrm{D}$ space.

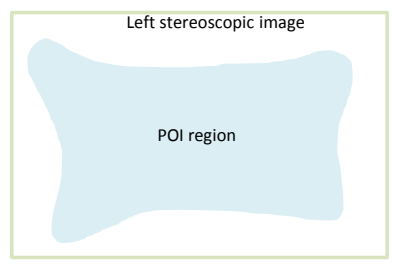

(a)

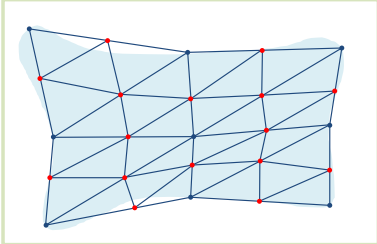

(c)

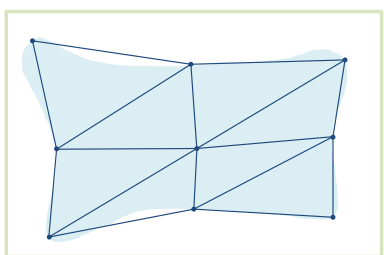

(b)

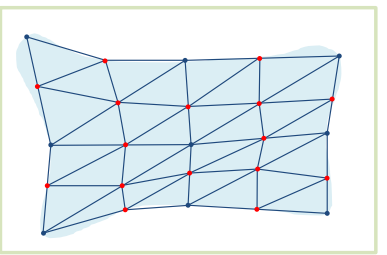

(d)

FIGURE 12: Generation of the 2D semi-regular mesh. (a) Left stereoscopic image and its POI region; (b) 2D base mesh; (c) Subdivision; (d) Displacement of the new vertices (red ones).

252 and Dussault, 2001). 


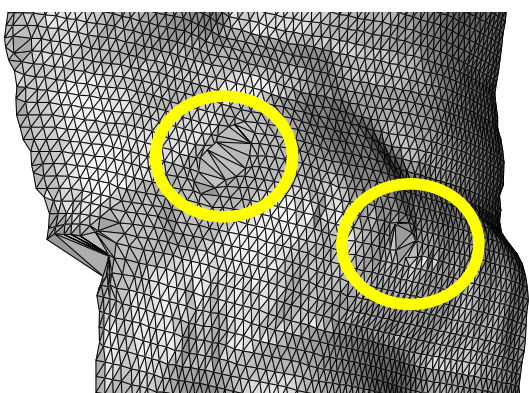

(a) Without our approximation.

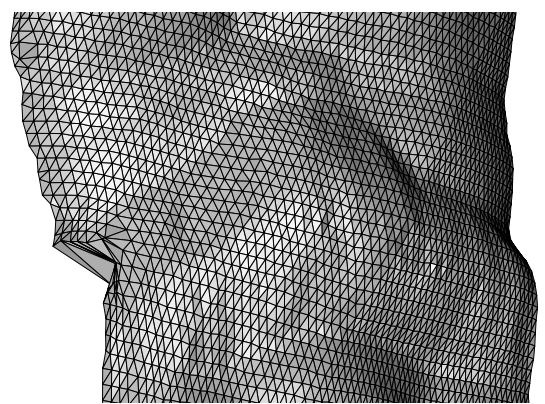

(b) With our approximation.

Figure 13: Technique proposed to fill the holes during the refinement.

\section{Experimental results}

\subsection{Visual results}

All our results are generated with a single pair of stereoscopic images obtained with a hand-held scanning system. Figure 14 gives an overview of our method on the model FACE.

From the stereoscopic images $(a)$, the POI region $(b)$ is defined, and the base mesh (resolution 0) is created $(c)$. Then, our coarse-to-fine approach generates several resolutions $(d, e, f)$. At resolution 5, our semi-regular reconstruction, with only $43 \mathrm{k}$ vertices, is already a good approximation of the original cloud of $250 \mathrm{k}$ points given by the stereoscopic system. This is promising in terms of both compactness and compression. Figure 14(g) also shows the textured semi-regular mesh, produced in a very simple way, with one stereoscopic image. No additional texturing technique is necessary as the connectivity of the semi-regular mesh is generated directly on the image domain. This is another great advantage of our approach.

\subsection{Uniform Vs adaptive sampling}

We now study the efficiency of our feature-preserving technique, and the difference in terms of triangle quality, between the meshes produced with the uniform/adaptive samplings during the creation of the base mesh (Section 3.3). 


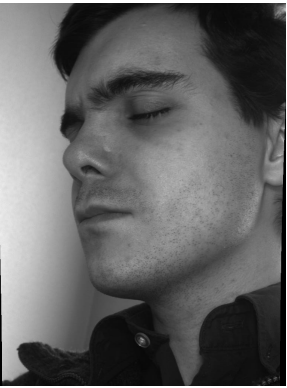

(a) Left image.

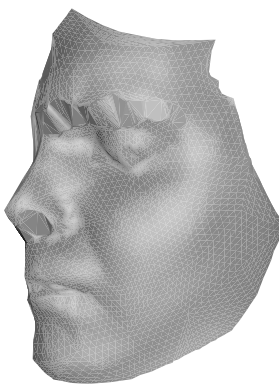

(e) Resolution 3

(2,745 vertices).

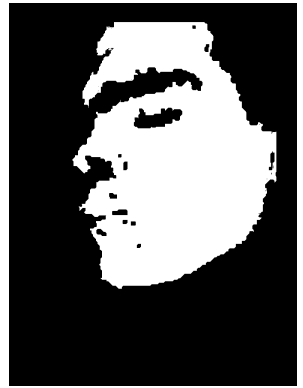

(b) POI region.

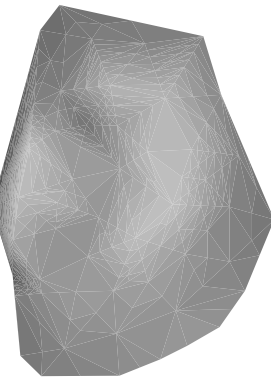

(c) Resolution 0

(50 vertices).

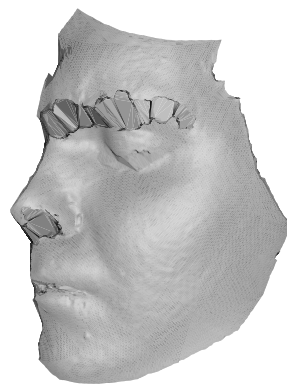

(f) Resolution 5

(43,233 vertices)

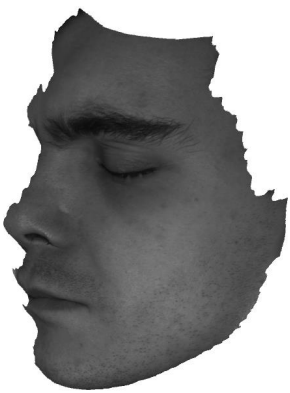

(g) Textured

semi-regular mesh.

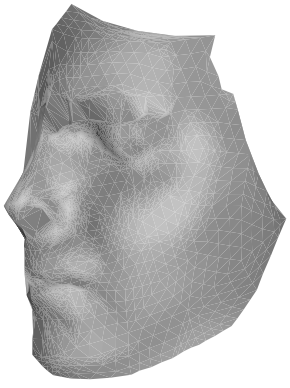

(d) Resolution 2

(701 vertices)

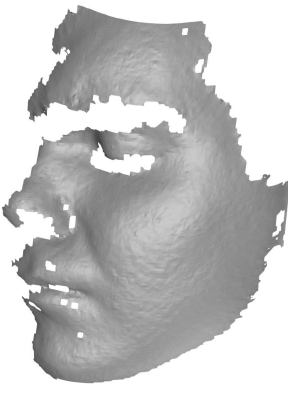

(h) Reference point cloud (249,767 pts).

Figure 14: Semi-regular reconstruction of the model FACE.

Figure 15 shows a reconstruction of a surface having sharp features called DoOR.

Subfigures $15(b),(c)$ and $(d)$ present the results with the uniform sampling, whereas subfigures $15(f),(g)$ and $h$ present the results with the adaptive sampling. We observe on the smooth shadings that the features are globally well preserved whatever the sampling. Some artifacts along them are visible, but they are due to the holes in the POI region that generate notches along features when the base mesh is created (these artifacts would be removed by improving the stereo matching in the scanning system). 

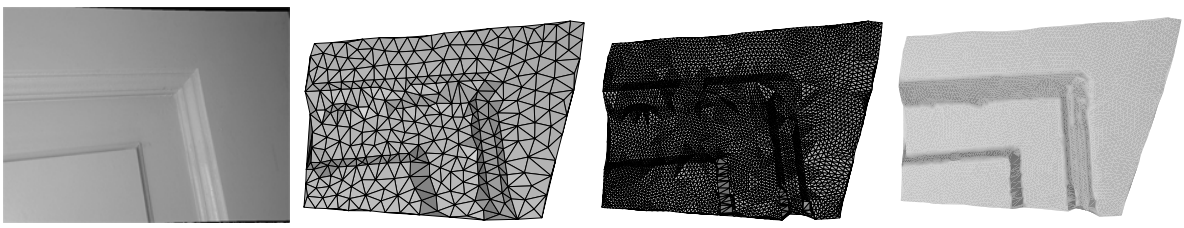

(a) Left image.

(b) Uniform base

(c) Resolution 2 mesh (300 vertices).

(4,575 vertices).
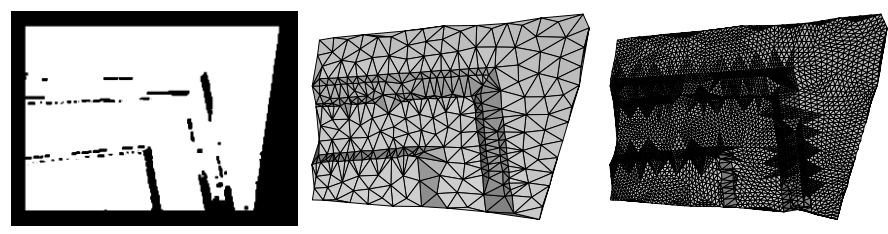

(d) Smooth shading.

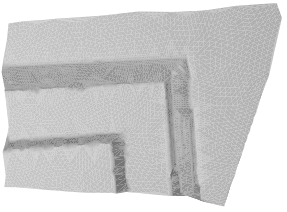

(h) Smooth shading.
(f) Adaptive base (g) Resolution 2
mesh (300 vertices). (4,599 vertices).
(e) POI region.

Figure 15: Preservation of the geometrical features of Door, with the uniform or the adaptive sampling.

However, we observe in subfigures $15(b)$ and $(f)$ that the adaptive sampling tends to better preserve the features from the lowest resolution. This result was expected, as the adaptive approach takes into account the curvature during the computation of the disks, leading to a dense sampling pattern along the geometrical features. The counterpart is that the sampling is globally less uniform, and the quality of the triangles is lower : the average minimum angle is $42.5^{\circ}$ and $37.5^{\circ}$ respectively for the uniform and the adaptive sampling.

Figure 16 gives an additional result on FACE : we see that the uniform sampling tends to provide a more isotropic mesh, and that the edges of the base mesh are less visible at high resolutions, which is advocated in case of smooth surfaces. On the database of five objects shown in Figure 17, the uniform sampling increases of $11 \%$ the average min angles. 

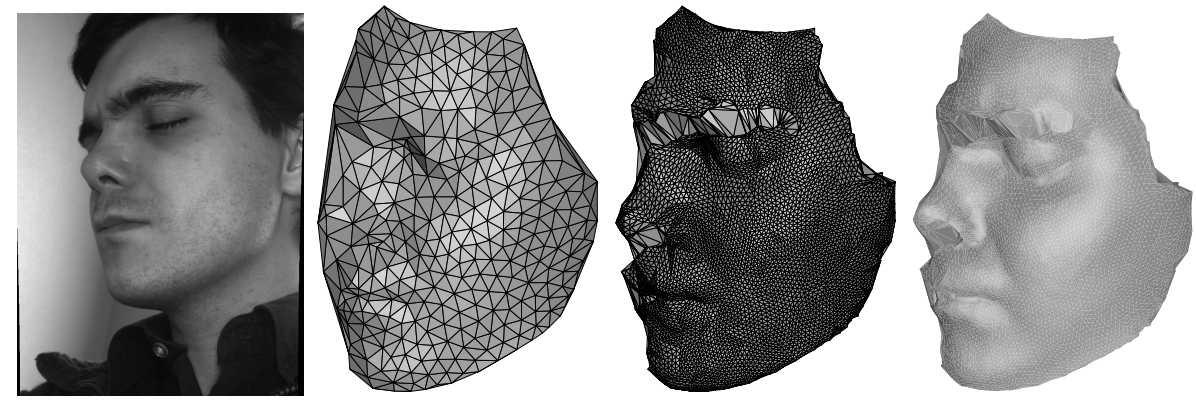

(a) Left image.

(b) Uniform mesh (300 vertices).

(4,611 vertices).
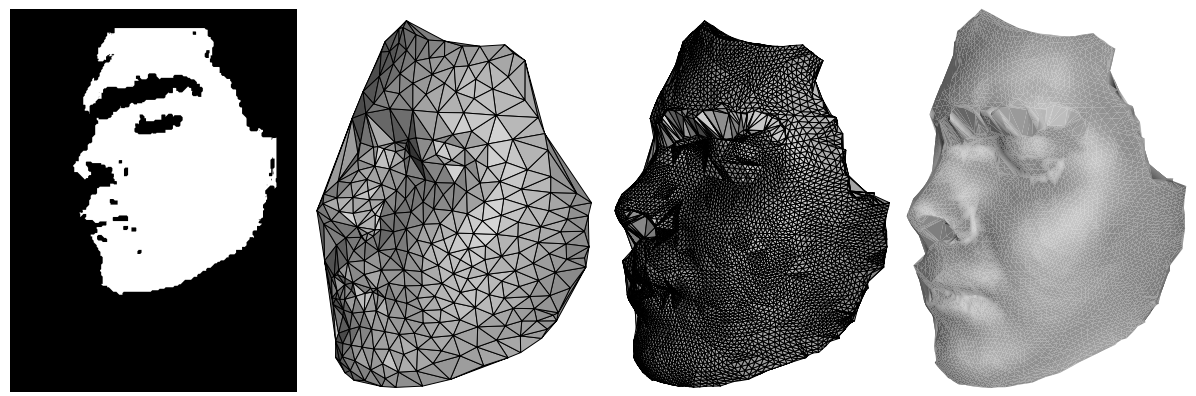

(e) POI region. (d) Smooth shading.

(h) Smooth shading. (f) Adaptive base mesh (300 vertices).

\begin{abstract}
(g) Resolution 2
\end{abstract} (4,629 vertices).

FIGURE 16: Difference of sampling quality obtained on FACE in function of uniform/adaptive sampling.

\subsection{Runtime}

We now evaluate the runtime of our semi-regular meshing on five surfaces shown in Figure 17. The results have been obtained with an Intel Core i3 CPU $2.30 \mathrm{GHz}$ processor, associated to a 4 GB RAM. 

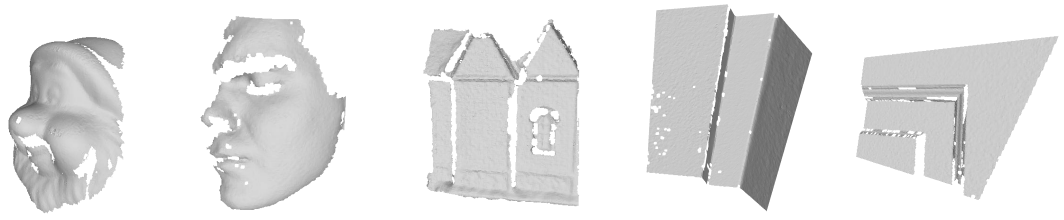

$\begin{array}{llcccc}(\mathrm{a}) & \text { Statue }(\mathrm{b}) & \text { FACE }(\mathrm{c}) & \text { House }(\mathrm{d}) & \text { Wall }(\mathrm{e}) & \text { Door } \\ (102,403 & (249,764 & (276,313 & (513,036 & (531,572 & \\ \text { points }) & \text { points }) & \text { points }) & \text { points }) & \text { points }) & \end{array}$

Figure 17: Database used to compute the runtimes of Figure 19 zoom of the graphic, where the Y-axis spans from 0 to 1.

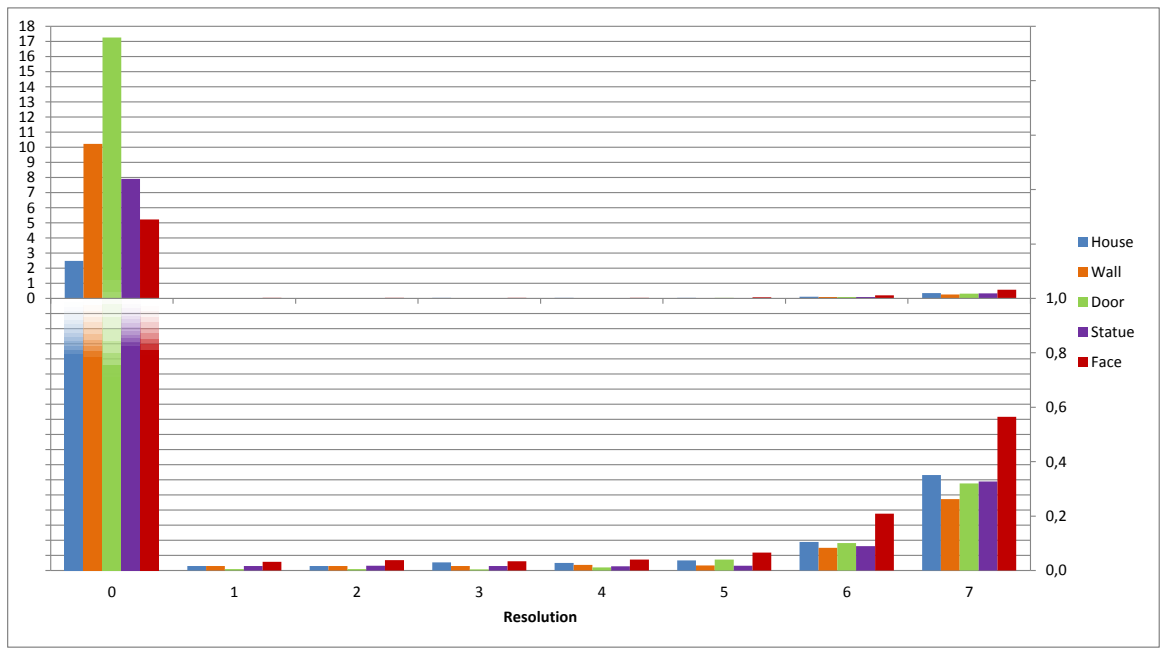

FiguRE 18: Runtime in seconds of our method, per resolution. The inferior part is a zoom of the superior one where the Y-axis spans from 0 to 1.

303

Figure 18 shows the runtime in function of the resolution, when the base mesh has 50 vertices, and eight resolutions. The inferior part of this figure is a

As expected, the most "greedy" resolution is the first one, during which the base mesh is generated (including the Poisson-disk sampling, the constrained 2D Voronoi relaxation, and the 2D Delaunay triangulation). The obtaining of the other resolutions is much faster, partly because theses steps have been pa- 

differences at each resolution for a same surface.

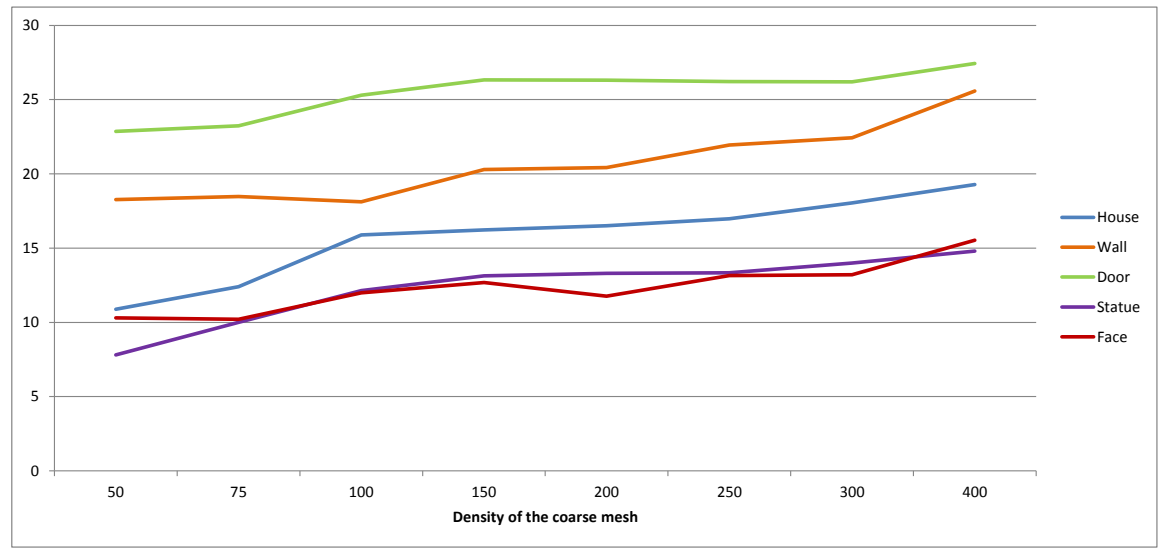

FiguRE 19: Runtimes (in sec.) of our method in function of the number of vertices of the base mesh.

rallelized on GPU. As a proof, the generation of the seventh resolution defined by 705,281 vertices $(528,768$ vertices are added) requires less than 0.6 seconds in the worst case. The higher total runtime to create our semi-regular mesh is around 17 seconds, for the model Door. Nevertheless, this remains very fast.

Now, Figure 19 shows the runtime in function of the base mesh density. For each surface, the curve is obtained by averaging the runtime of five tests. Indeed, our algorithm is not deterministic (because of the dart throwing), and the relaxation time depends on the initial sampling. So, we can obtain slightly

$$
\begin{aligned}
& \text { We observe the linearity of the total runtime with respect to the number of } \\
& \text { vertices of the base mesh. The differences between the models is due to the ori- } \\
& \text { ginal point cloud density (ranging from 102, } 403 \text { points for STATUE to } 531,572 \\
& \text { points for Door). The curvature of the scanned surface also influences the run- } \\
& \text { time. For instance, WALL contains around } 18 k \text { points less than Door, but it } \\
& \text { contains much more pixels classified as sharp features. Finally, the mean sam- } \\
& \text { pling runtime is slower : } 5.70 \text { seconds for WALL, while it amounts to } 10.90 \\
& \text { seconds for Door. }
\end{aligned}
$$




\subsection{Comparison with the classical pipeline}

We now compare our direct semi-regular meshing with the classical acquisition pipeline to get a semi-regular mesh (point cloud generation $\rightarrow$ triangulation $\rightarrow$ semi-regular remeshing). We use a Voronoi-based triangulation technique to generate the original irregular reference mesh $M_{\text {ori }}$ from the point cloud provided by our stereoscopic system. This irregular mesh is then remeshed semiregularly either with the SDK SmartMesh based on the patent Fonteles et al. (2014) and developed by the company (Cintoo3D) (a free trial is available on the website), or with Trireme (Guskov, 2007). To our knowledge, they are the only semi-regular remeshing techniques available on the web. Unfortunately, we found out that Trireme is not a suitable tool to remesh our data. Indeed, we could not produce any semi-regular meshes without severe degeneracies and outlier triangles. In the contrary, SmartMesh always provides manifold semi-regular meshes, in particular because it does not use any parameterization, unlike Trireme. Consequently, we only compare the reconstruction errors and runtimes relative to our semi-regular meshing and to SmartMesh.

We first compute the symmetric root mean square distance between the set of vertices of our semi-regular meshes, and the set of vertices of $M_{\text {ori }}$ (the original point cloud provided by the acquisition system). It permits to assess the fidelity of our sampling to the reference point cloud. The same distance is calculated with the set of vertices of the semi-regular meshes produced by SmartMesh. Figure 20 shows the evolutions of these distances depending on the resolutions : the $\mathrm{X}$-axis indicates the associated number of points. We observe that our method presents lower distances than SmartMesh. It was expected as our method is approximating, contrary to SmartMesh that is interpolating (it optimizes the positions of the vertices such as its semi-regular mesh is close to the reference mesh). Our method has the advantage to determine the majority of vertices among the original point cloud, as the vertices are selected via the pixels of the POI region in the image domain. The only vertices that do not exist in the original point cloud are associated to pixels selected outside the POI region 

the geometry of the initial surface.

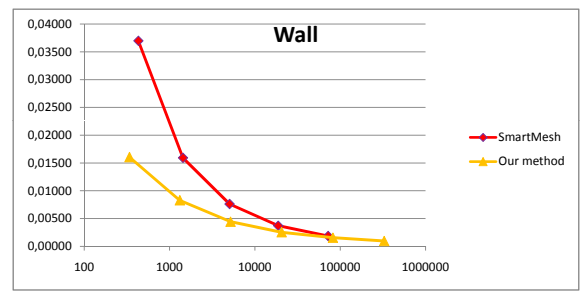

(a) WALL

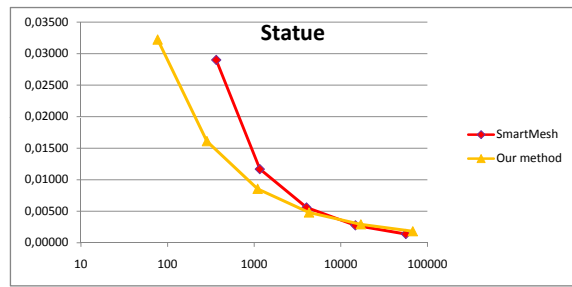

(c) Statue

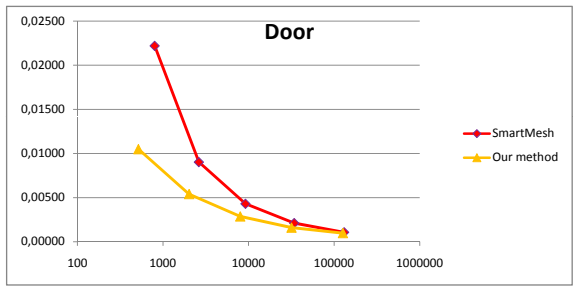

(b) Door

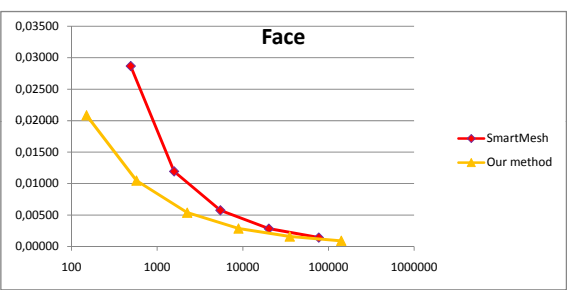

(d) FACE

Figure 20: Comparison of the geometry sampling obtained with our method and with SmartMesh (Cintoo3D), depending on the vertex density of the semi-regular meshes.

We now assess the fidelity of our semi-regular meshes with respect to the reference mesh $M_{o r i}$. To achieve this goal, we compute the symmetric Root Mean Square (RMS) distance between $M_{\text {ori }}$ and our semi-regular meshes $M_{s r}$ (normalized by the diagonal length of the bounding box), which is widespread used in the state-of-the-art (Payan et al., 2015). However, in our context, this measure is not suited. Indeed, as explained in section 3.4, our method fills the holes, in order to make the texturing easier and to enhance the mesh quality. As a consequence, when measuring the symmetric RMS distances between our semi-regular meshes and the reference irregular mesh $M_{\text {ori }}$, the distances between the triangles filling the holes and the original surface are inevitably high. It severely corrupts the comparison with SmartMesh, as this latter has been initially developed to preserve the potential borders of a surface and consequently the holes. So, to make fairly comparisons, we compute the asymmetric RMS 


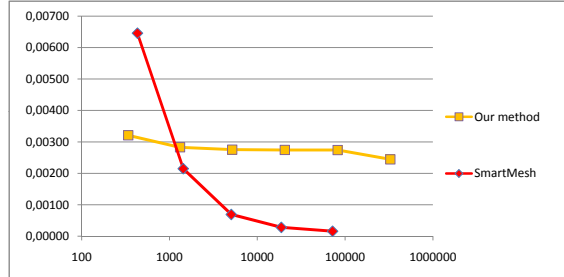

(a) WALL

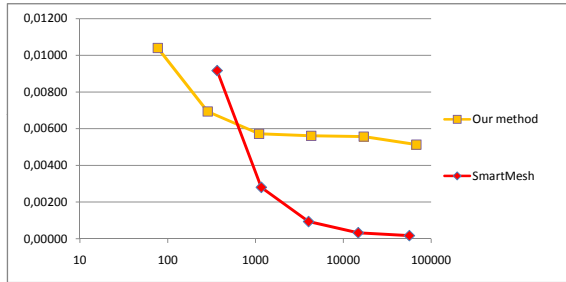

(c) Statue

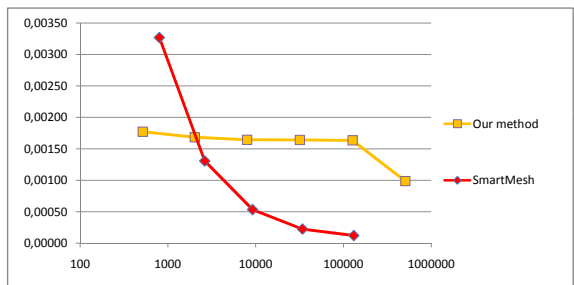

(b) Door

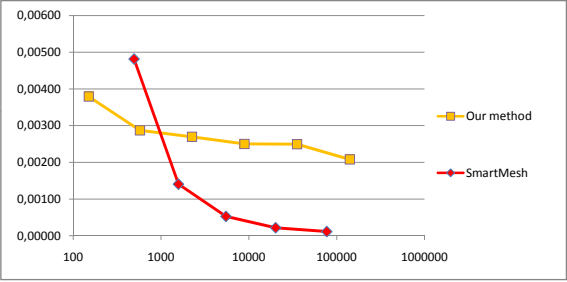

(d) FACE

FIGURE 21: addAsymmetric RMS distance $R M S\left(M_{o r i} \rightarrow M_{s r}\right)$ obtained with our method and with SmartMesh (Cintoo3D) in function of the resolution.

On the other hand, our algorithm is direct and thus significantly faster than SmartMesh. The runtime comparison is summarized in table1. SmartMesh indeed takes several minutes to produce the semi-regular meshes : from 2 to 7 minutes in function of the data, excluding the triangulation time, whereas our 

pipeline is a promising alternative to the classical one.

\begin{tabular}{|c|c|c|c|c|}
\hline Models & Wall & Door & Statue & Face \\
\hline Base mesh density & 431 & 800 & 365 & 492 \\
\hline SmartMesh & $\geq 3$ min. & $\geq 7$ min. & $\geq 2$ min. & $\geq 6$ min. \\
\hline Our method & 19.1 sec. & 27.7 sec. & 10.1 sec. & 10.3 sec. \\
\hline
\end{tabular}

TABLE 1: Runtime comparison in seconds between our method and SmartMesh (Cintoo3D), to generate 5 resolutions.

388

method needs always less than one minute. Finally, it shows that the proposed

\section{Conclusion and perspectives}

In this paper we proposed an alternative to the fastidious pipeline to get semi-regular meshes from physical objects. The idea is to generate semi-regular meshes directly from the stereoscopic images acquired with a hand-held stereo acquisition system. The key idea of our work is that the stereoscopic images can be considered as a parameterization of the acquired surface. Therefore, our reconstruction method processes the data as much as possible into the image domain, before embedding the surface in the 3D space.

The first contribution is an original sampling that creates a base mesh of the scanned surface. We show that the Poisson-disk sampling developed by (Peyrot et al. 2013) can be extended to a stereoscopic system, while retrieving the 3D information necessary to preserve features all along the process. This allows to take into account the surface geometry, although the sampling is realized on the stereoscopic images. The second contribution concerns our coarse-to-fine approach that allows to get a semi-regular mesh preserving the geometrical features as output of our acquisition system, by working mainly in the image domain.

Our pipeline could be easily included into any stereoscopic acquisition system. It also has the advantage to create semi-regular output that can be directly 
textured with the stereoscopic image, and is also much more faster and convenient than the classical pipeline.

However, a lot of improvements remains possible. For instance, the runtime of our algorithm can be improved as some parts are implemented on CPU. It would be interesting to investigate parallel algorithms for all the stages, to allow quasi real-time reconstructions. We could also investigate new means to improve the shape fidelity, in order to be competitive with the semi-regular remeshing techniques. Another promising improvement would be to manage several views. Indeed, our current algorithm handles only one view, and thus only a part of the scanned object can be reconstructed. It would be relevant to study, for instance, mosaicing techniques, widespread in photogrammetry, to generate a large POI region representing the whole parameterized object, and thus to output a complete semi-regular representation of a physical object.

Acknowledgements This work was supported by a grant from Région Provence Alpes Côte d'Azur in France.

\section{Bibliography}

Q. Chen and H. Prautzsch. General midpoint subdivision. CoRR, abs/1208.3794, 2012.

Cintoo3D. SDK smartmesh. http://www. cintoo3D.com.

E. W. Dijkstra. A note on two problems in connexion with graphs. Numerische Mathematik, 1 :269-271, 1959.

R. Egli and J.-P. Dussault, editors. Technique butterfly généralisée, Dijon, France, p. 133-136, 2001. Actes de Compression et Représentation des Signaux Audiovisuels. 
L. Hidd Fonteles, A. Meftah, M. Antonini, and F. Payan. Method, system and computer program product for 3d objects graphical representation. FR-13, n 4152728.3-1502, 2014, CNRS et Universit de Nice - Sophia Antipolis, 2014.

I. Guskov. Manifold-based approach to semi-regular remeshing. Graphical Models, 69(1) :1-18, 2007.

R. I. Hartley and A. Zisserman. Multiple View Geometry in Computer Vision. Cambridge University Press, second edition, 2004.

E. C. Hernandez, G. Vogiatzis, and R. Cipolla. Multiview photometric stereo. IEEE Trans. Pattern Anal. Mach. Intell., 30(3), March 2008.

S. P. Lloyd. Least squares quantization in pcm. IEEE Transactions on Information Theory, 28 :129-137, 1982.

M. Lounsbery, T. D. DeRose, and J. Warren. Multiresolution analysis for surfaces of arbitrary topological type. ACM Trans. Graph., 16(1), January 1997.

A. Munshi, B.-R. Gaster, T.-G. Mattson, J. Fung, and D. Ginsburg. OpenCL Programming Guide. Prentice Hall, 2011.

J. Park, S. N. Sinha, Y. Matsushita, Y.-W. Tai, and I. S. Kweon. Multiview photometric stereo using planar mesh parameterization. Computer Vision, IEEE International Conference on, 0, a.

M. K. Park, S. J. Lee, and K. H. Lee. b.

F. Payan and M. Antonini. Mean square error approximation for wavelet-based semiregular mesh compression. Transactions on Visualization and Computer Graphics (TVCG), 12, 2006.

F. Payan, C. Roudet, and B. Sauvage. Semi-regular triangle remeshing : a comprehensive study. Computer Graphics Forum, 34 :86-102, February 2015.

J.-L. Peyrot, F. Payan, and M. Antonini. Feature-preserving Direct Blue Noise Sampling for Surface Meshes. In Eurographics (Short Papers), pages 9-12, 2013. 
J.-L. Peyrot, F. Payan, N. Ruchaud, and M. Antonini. Stereo reconstruction of semiregular meshes, and multiresolution analysis for automatic detection of dents on surfaces. In Proceedings of IEEE International Conference in Image Processing (ICIP), Paris, France, october 2014.

J.-L. Peyrot, F. Payan, and M. Antonini. Direct blue noise resampling of meshes of arbitrary topology. The Visual Computer, 31 :1365-1381, september 2015.

N. Pietroni, M. Tarini, O. Sorkine, and D. Zorin. Global parametrization of range image sets. ACM Trans. Graph., 30(6), December 2011.

G. Rong, T.-S. Tan, T.-T. Cao, and Stephanus. In Eric Haines and Morgan McGuire, editors, SI3D.

D. Scharstein and R. Szeliski. A taxonomy and evaluation of dense two-frame stereo correspondence algorithms. Int. J. Comput. Vision, 47(1-3) :7-42, April 2002. ISSN 0920-5691.

S. M. Seitz, B. Curless, J. Diebel, D. Scharstein, and R. Szeliski. A comparison and evaluation of multi-view stereo reconstruction algorithms. In Proceedings of the 2006 IEEE Computer Society Conference on Computer Vision and Pattern Recognition - Volume 1, CVPR '06, 2006.

N. Snavely, S. M. Seitz, and R. Szeliski. Photo tourism : Exploring photo collections in 3d. ACM Trans. Graph., 25(3) :835-846, July 2006.

T. Y. Zhang and C. Y. Suen. A fast parallel algorithm for thinning digital patterns. Commun. ACM, 27(3), March 1984.

K. Zhou, J. Synder, B. Guo, and H.-Y. Shum. Iso-charts : Stretch-driven mesh parameterization using spectral analysis. Eurographics, July 2004. 